\title{
Combination of heliox and CPAP without a ventilator: bench test and clinical observations
}

\author{
doi: $10.1017 / \mathrm{S} 0265021507000695$
}

\section{EDITOR:}

Failure to restore an adequate airway in patients with acute upper airway obstruction can rapidly lead to hypoxic injury or even death. Helium-oxygen mixtures (heliox) are less dense than air-oxygen mixtures and can improve the flow of gas through partially obstructed airways. Since Barach described this principle 70 years ago, heliox has at times been applied in spontaneously breathing patients with acute upper airways obstruction [1]. Heliox has also been combined with (non-) invasive mechanical ventilation, often with specifically adapted apparatus [2]. However, the correct function of ventilators relies on the precise measurement of flow, pressure, temperature and oxygen fraction for which the necessary sensors are calibrated for use with air-oxygen. The unique physical properties of heliox have an important impact on all these measurements. Thus the correct application of heliox in ventilators is far from trivial [3]. This technical problem becomes especially relevant under emergency circumstances.

Continuous positive airway pressure (CPAP) by face mask can reduce upper airway collapse [4] and the combination of mask CPAP and heliox might be useful. The Boussignac continuous positive airway pressure (BCPAP) system (Vygon, 95440 Ecouen, France) for mask CPAP is a simple and lightweight $(10 \mathrm{~g})$ disposable plastic device without moving parts [5]. This device may thus avoid the problems with ventilators and heliox.

In a bench test, we evaluated whether heliox works with the BCPAP system and how much more heliox flow was needed compared to oxygen to achieve similar pressures. We then evaluated the effect of this combination in selected patients with acute upper airways obstruction. We chose a

Correspondence to: Willem Dieperink, Surgical Intensive Care Unit, University Medical Center Groningen, University of Groningen, P.O. Box 30.001, 9700 RB Groningen, The Netherlands. E-mail: w.dieperink@chir.umcg.nl; Tel: +31 50 3612306; Fax: +31503619986

Accepted for publication 24 March 2007 EJA 4152

First published online 20 June 2007 helium-oxygen mixture of $60 / 40$ to ensure a sufficient oxygen delivery under all circumstances.

For the bench test we used 10 randomly selected, commercially available BCPAP systems and an analyser specifically designed to measure pressure or flow for helium-oxygen mixtures (VT Plus, Fluke Biomedical, Carson City, NV, USA). Since the variable orifice flow meters mounted on the heliox cylinders were oxygen-calibrated, we first recalibrated these meters by comparing the indicated flow of oxygen or heliox with the true flow as measured with the VTplus analyser. This was done 10 times for each of the following levels of indicated flow: 5, 10, 15, 20, 25 and $30 \mathrm{Lmin}^{-1}$. The observed relation between indicated and actual flow was also compared to the theoretically predicted flow [6]. After this flowcalibration, we then measured the pressures generated by the BCPAP system for the various indicated flows (Fig. 1). The measured flow rates (true flow) of the variable orifice flow meters showed a $25 \pm 6 \%$ (SD) higher flow for heliox compared to oxygen. When we calculated the predicted actual flow for the variable orifice flow meters for heliox $60 / 40$ based on

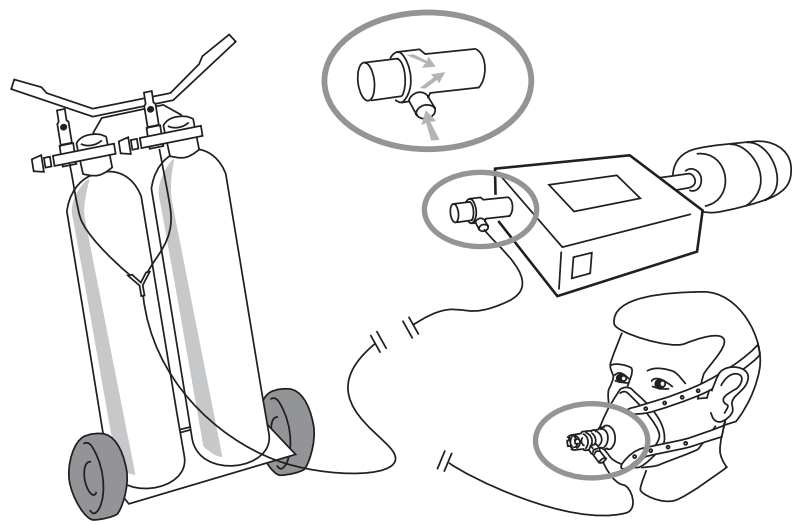

Figure 1.

In a bench test (upper right), the static pressures generated by the Boussignac continuous positive airway pressure (BCPAP) system for various flows of heliox 60/40 were measured in a closed system. For this purpose, the patient side of the BCPAP system was connected to a pressure analyser. In patients, the BCPAP system was connected to a face mask. 
the gas laws and assuming fully turbulent conditions with densities $\left(\rho=\right.$ rho) of 1.33 and $0.17 \mathrm{~kg} \mathrm{~m}^{-3}$ for oxygen and heliox, respectively [6]: $\left(\rho \mathrm{O}_{2} / \rho\right.$ Heliox $60 / 40)^{0.5} \approx(1.33 / 0.63)^{0.5}$, the theoretically predicted actual flow was $145 \%$ of the indicated flow. Thus the behaviour of the variable orifice flow meter with heliox was not in accordance with the assumptions of the simplified gas law.

Flow-pressure measurements were performed at six levels of flow indicated by the orifice flow meters. At every flow level, pressure was measured 10 times. With regard to the generated pressure by the BCPAP system, both for oxygen and for heliox, an approximately linear flow-pressure relation was observed. Compared to oxygen, a true heliox flow of 178\% (95\% CI: 168-188\%) was needed to achieve similar levels of CPAP.

For rapid transport to any patient in our hospital with acute upper airway obstruction, we put the heliox cylinders and the BCPAP system, as well as conventional non-rebreathing masks, on a small trolley (Fig. 1). Over a 2-yr period, seven adult patients (aged 53-83yr) and one infant aged 3 months with life-threatening obstruction and various degrees of inspiratory stridor were treated. Causes of the stridor included laryngeal cancer, vocal cord palsy, post extubation stridor or hygroma. Heliox and BCPAP were administered for a mean (SD) duration of $83 \pm 42$ (range 60-180) min. We used indicated flows from 20 to $30 \mathrm{~L} \mathrm{~min}^{-1}$, corresponding to true flow of $25-36 \mathrm{~L} \mathrm{~min}^{-1}$ and pressures of $6-8 \mathrm{~cm}$ of $\mathrm{H}_{2} \mathrm{O}$. In all cases stridor typically decreased or disappeared within a few minutes. In four patients a definitive airway was established after intubation with an endotracheal tube and in four cases with a tracheostomy. In one patient we successfully performed flexible intubation through the BCPAP system.

We showed that heliox could effectively generate pressure in the BCPAP system. The simplicity of this system with no moving parts allowed us to put it on a trolley that could be brought to patients. The application of heliox and BCPAP was effective in creating clinical stabilization allowing time to create a final secure airway. Acute upper airway obstruction is often a life-threatening situation. Under such circumstances, establishing a safe definitive airway is crucial, but the procedure itself is one of the most dangerous actions in medicine. It should be stressed that neither CPAP nor heliox nor CPAP and heliox combined are intended to solve the airway obstruction, but to immediately relieve the work of breathing and buy some time. In the meantime, the precise cause of respiratory failure may be identified and an experienced team can prepare to establish a definitive airway. The considerable cost of heliox [1] is another reason to limit the duration of treatment with heliox. There are several reports of clinical application of heliox and non-invasive ventilation [2]. It is known that non-invasive ventilation and also CPAP decrease the work of breathing in patients with upper airway obstruction [4]. Heliox also decreased the work of breathing in these patients. Obviously, CPAP alone or heliox without CPAP given by venturi mask or non-rebreathing mask may be the easiest and best solution for many patients. We assumed that combining heliox and BCPAP might have additive effects. Recently, a study showed the effectiveness of the combination nasal CPAP and heliox in infants [7]. To our knowledge there are no reports in adults of combining heliox specifically with CPAP devices, including the BCPAP system. The unique open design of the BCPAP system makes bronchoscopy [5] or even endotracheal intubation, through the system, possible with no loss of CPAP. As the true heliox flow differs both from the actual and from the theoretically predicted flow, recalibration of flow meters is mandatory, especially since the gas cylinders empty much faster than might otherwise be expected.

The simple BCPAP system circumvents many of the disadvantages associated with the use of heliox with mechanical valves or ventilators. We think the BCPAP heliox combination may be of use as a bridging therapy in selected patients with acute upper airway obstruction. Prospective studies are needed before a final recommendation can be made about the benefit of this therapy.

W. Dieperink

Surgical Intensive Care Unit

University Medical Center Groningen University of Groningen Hanzeplein Groningen, The Netherlands

J. A. Knol Department of Anaesthesiology Tjongerschans Hospital Thialfweg

Heerenveen, The Netherlands

H. J. Boersma

Care Service Medical Instrumentation

University Medical Center Groningen University of Groningen Hanzeplein Groningen, The Netherlands

G. B. Eindhoven, L. P. H. J. Aarts Department of Anaesthesiology

University Medical Center Groningen University of Groningen 


\author{
Hanzeplein \\ Groningen, The Netherlands \\ J. F. Goorhuis \\ Department of Paediatrics \\ Division of Intensive Care \\ University Medical Center Groningen \\ University of Groningen \\ Hanzeplein \\ Groningen, The Netherlands \\ M. W. N. Nijsten \\ Department of Surgery \\ University Medical Center Groningen \\ University of Groningen \\ Hanzeplein \\ Groningen, The Netherlands
}

\section{References}

1. McGee DL, Wald DA, Hinchliffe S. Helium-oxygen therapy in the emergency department. J Emerg Med 1997; 15: 291-296.
2. Jaber S, Fodil R, Carlucci A et al. Noninvasive ventilation with helium-oxygen in acute exacerbations of chronic obstructive pulmonary disease. Am J Respir Crit Care Med 2000; 161: 1191-1200.

3. Tassaux D, Jolliet P, Thouret JM, Roeseler J, Dorne R, Chevrolet JC. Calibration of seven ICU ventilators for mechanical ventilation with helium-oxygen mixtures. Am J Respir Crit Care Med 1999; 16: 22-32.

4. Visvanathan T, Kluger MT, Webb RK, Westhorpe RN. Crisis management during anaesthesia: obstruction of the natural airway. Qual Saf Health Care 2005; 14: e2.

5. Maitre B, Jaber S, Maggiore SM et al. Continuous positive airway pressure during fiberoptic bronchoscopy in hypoxemic patients. A randomized double-blind study using a new device. Am J Respir Crit Care Med 2000; 162: 1063-1067.

6. Papamoschou D. Theoretical validation of the respiratory benefits of helium-oxygen mixtures. Respir Physiol 1995; 99: 183-190.

7. Martinon-Torres F, Rodriguez-Núñez A, MartinonSánchez JM. Nasal continuous positive airway pressure with heliox in infants with acute bronchiolitis. Respir Med 2006; 100: 1458-1462.

\section{Renal impact of fluid management with colloids}

doi: $10.1017 / \mathrm{S} 0265021507000774$

\section{EDITOR:}

We have read Dr Davidson's article on volume replacement using colloids with great interest [1]. The author made great efforts to analyse the influence of different colloids on kidney function. The author's conclusion that 'Colloids display important differences in their actions on the kidneys' is precise and well balanced. Unfortunately, the author did not distinguish the effects of the different hydroxyethylstarch (HES) preparations on renal function with the same accuracy. He cited several studies and concluded that 'Undesirable renal effects are common to all available HES solutions ... '. Several articles were cited, however, without any cautious comments of their value: the multicentre study by Schortgen and colleagues [2] is one such example. This study has already been criticized by others $[3,4]$ showing that patients treated with $6 \%$ HES 200/0.62 were not different from a gelatin-treated group with regard to the need for renal-replacement

Correspondence to: Joachim Boldt, Department of Anesthesiology and Intensive Care Medicine, Klinikum der Stadt Ludwigshafen, Bremserstr. 79, D-67063 Ludwigshafen, Germany. E-mail: BoldtJ@gmx.net; Tel: +49 621503 3000; Fax: +496215033024

Accepted for publication 16 November 2006 EJA 4238

First published online 20 June 2007 therapy - mortality was also not different; there was even no trend for increased mortality in the HES 200/0.62-treated group. The definition of acute renal failure (ARF) was based only on the creatinine levels. Unfortunately, these were already higher in the HES-treated group at baseline compared to the gelatin-treated group, suggesting that renal function was perhaps quite different already at the start of the study.

Most importantly, however, we feel urged to comment on some of the author's statements because he is referring to some of our articles - but with entirely different conclusions from those that we reached. In one of our studies focussing on the effects of HES 130/0.4 on kidney function in elderly patients undergoing cardiac surgery using cardiopulmonary bypass [5], we used the gelatin-treated group as our control group because there are no well-performed studies showing increased incidence of ARF requiring haemodialysis after the use of gelatins. Dr Davidson confirmed his conclusion that 'Renal dysfunction was documented in HES 130/ 0.4 recipients by all four markers (of impaired kidney integrity)' by showing our graphs. In our study, we came to a completely different conclusion. All measured kidney-specific proteins increased in 